\title{
Proposal of Automatic Dust Catcher Robot of Clothes
}

\author{
Natsuki Nakamoto ${ }^{a}$, Yuhki Kitazono ${ }^{\mathrm{a},{ }^{*}}$

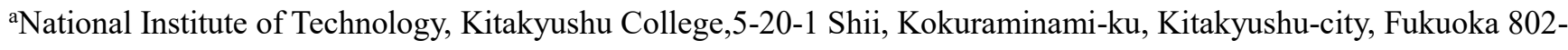 \\ 0985, Japan \\ *Corresponding Author: kitazono@kct.ac.jp
}

\begin{abstract}
People remove dust and animal hair on clothes by themselves every day. It consumes a lot of effort and time. Therefore, we propose a robot that automatically removes dust from clothes hanging on hangers with a brush. This robot uses two brushes. At first, two brushes pinch the top part of both sides of clothes hanging on the hanger. Next, move the brush from top to bottom to remove dust. In addition, by moving the brush sideways and repeating the action of removing dust, dust can be removed from the entire clothes. This robot can save labor every day.
\end{abstract}

Keywords: dust, clothes, brush.

\section{Introduction}

House dust, mites, and animals can cause allergies ${ }^{(1)(2)}$. It is dangerous to come in contact with allergy sufferers with these substances on your clothes ${ }^{(3)}$. Therefore, it is necessary to remove dust and other particles from clothes when there is a possibility of contacting with people.

Also, appearance is a quite important point in giving a positive impression on others. According to the "Questionnaire on Marriage Activities" conducted in 2019 by Partner Agent, a dating agency operated by Tameny Corporation shows, $54.7 \%$ of 320 men and women aged 20 39 answered that cleanliness is an important factor in what they look for in a marriage partner ${ }^{(4)}$.

Wearing clean clothes is one of the things we need to do to maintain good hygiene for ourselves and others, and to build good relationships with others. For this purpose, it is necessary to wash, iron out wrinkles, and remove dust from clothes. In this paper, we focused on the task of removing dust from clothes. Since the average member of household in Japan is two people, we consider the amount of dust generated at this time ${ }^{(5)}$. According to "On the generation of

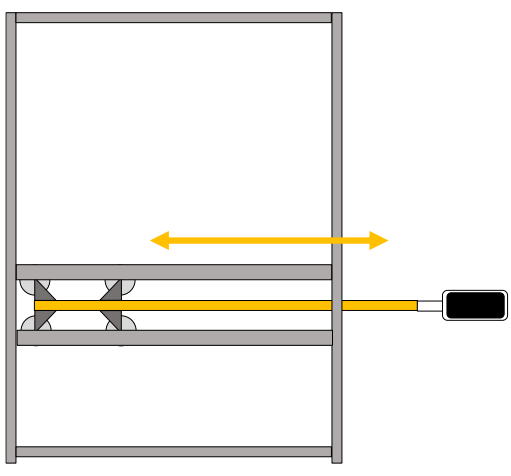

(a) Front view.

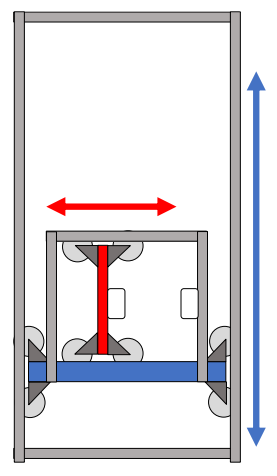

(b) Side view.
Fig. 1. Robot overview.

dust in rooms", a collection of reports by the Architectural Institute of Japan in 1966, the dust generation rate when two people are in one room is $2.44 \times 10^{-3}\left(\mathrm{~m}^{3} / \mathrm{s}\right)^{(6)}$. Furthermore, according to the national survey of the Pet Food Association's in 2020, 6.8 million households have 8.49 million dogs and 5.51 million households have 9.64 million cats $^{(7)}$. One Labrador retriever sheds 238 hairs per 30 minutes, which is 11424 hairs a day ${ }^{(8)}$. If you keep animals such as dogs and cats indoors, a lot of animal hair will stick to your clothes in addition to dust. Dust and animal hair on clothes are removed by using a roll of adhesive tape, a special brush, and other useful items. However, this needs to be done manually, which can be a big burden if you have a lot of clothes or if you have a dog or cat.

In this paper, we propose a robot automatically to remove dust from clothes to reduce the burden at home. This robot can automatically remove dust from both sides of clothes by holding the clothes on the hanger between two dust-removing brushes and moving them from the top to the bottom. The brushes can also be moved sideways to remove dust from the entire clothes. By using this robot, you can save time and effort every morning. 


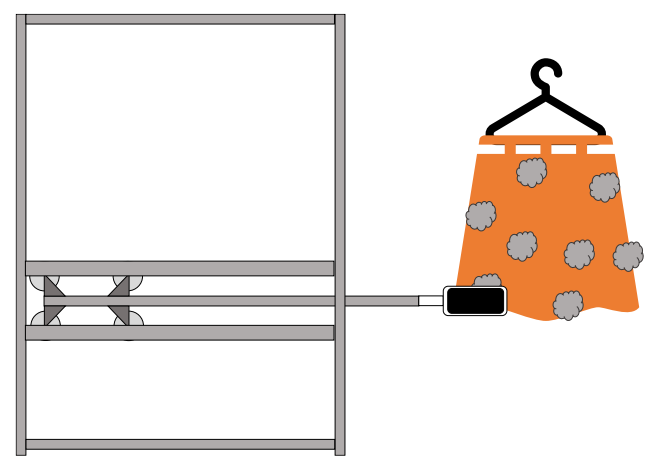

(a) Start

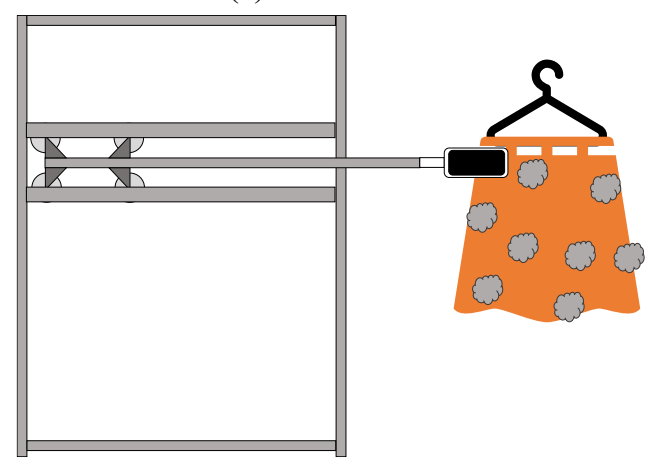

(b) Step 1 .

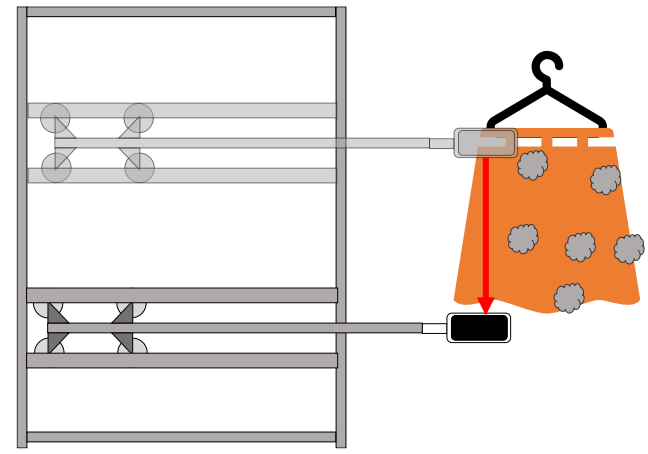

(c) Step 2 .

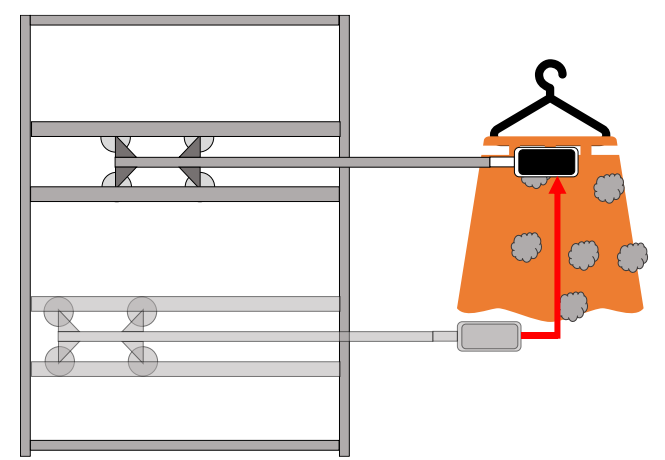

(d) Step 3 .

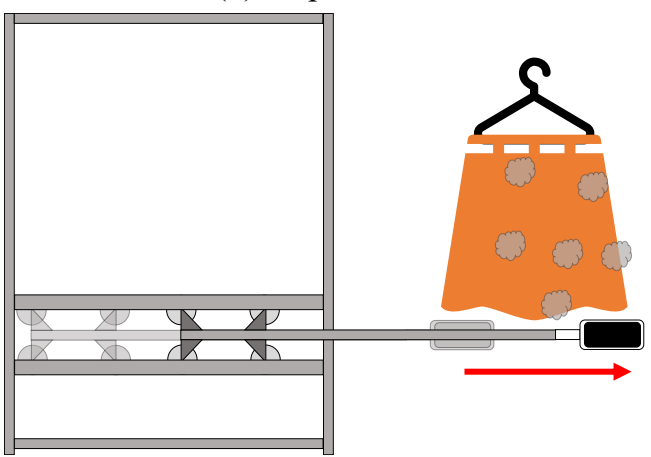

(e) Step 4 .

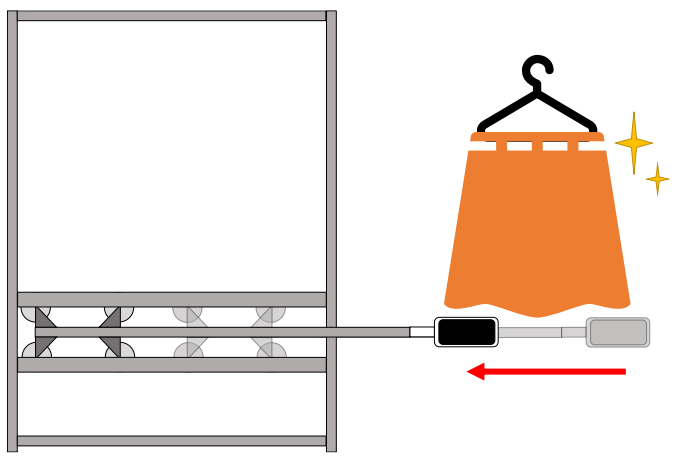

(f) Step 5 .

Fig. 2. Operating procedure.

\section{Automatic Dust Catcher Robot of Clothes}

A schematic diagram of the robot is shown in Fig. 1. This robot can hold a clothes between two brushes and move them up and down to remove dust from the clothes. From Fig. 1 , each moving part corresponds to the following axis: red for holding the clothes between the brushes, blue for moving the brushes up and down to remove dust from the clothes, and yellow for extending the brushes to remove dust from the whole clothes. The moving parts move in the direction of the arrows, respectively. By repeating these motions, the entire clothes can be dusted.

The user hangs the clothes to be dusted on the hanger rack so that the two brushes pinch the front of the clothes as seen from the robot. Press the start button to start the robot's operation. Once it starts, the robot repeatedly moves the three axes to remove dust from the clothes. When the robot finishes dusting the entire clothes, it shrinks the brushes to finish the operation.

The above procedure is explained concretely with illustrations. First, set the clothes as shown in Fig. 2(a). When the operation starts, the robot brings the brush to the top of the clothes as shown in Fig. 2(b). When the brush reaches the top of the clothes as shown in Fig. 2(c), the robot grips the clothes between the brushes and lowers the brush down to remove dust from the clothes. Then, as shown in Fig. 2(d), the motions of releasing the clothes from the brush, stretching the brush to the side, and raising the brush are performed in that order. At this point, extend the brushes one by one. As shown in Figs. 2(e) and 2(f), the above operation is repeated, and when the dust is removed from the entire 

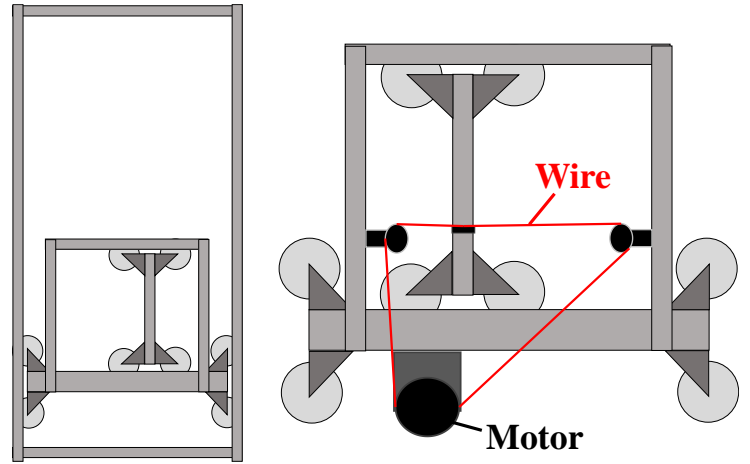

(a) Wire and motor.
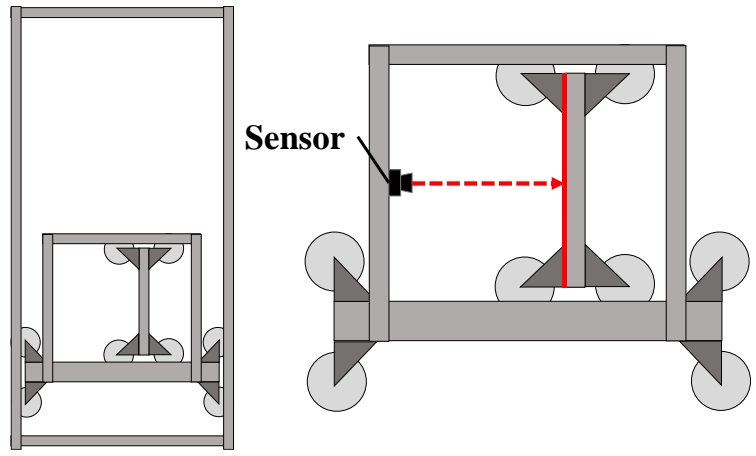

(b) Sensor.

Fig. 3. Hold clothes.

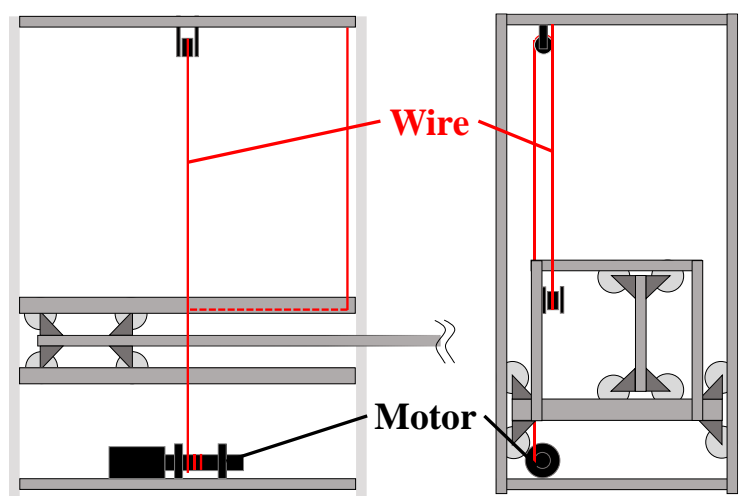

(a) Wire and motor.

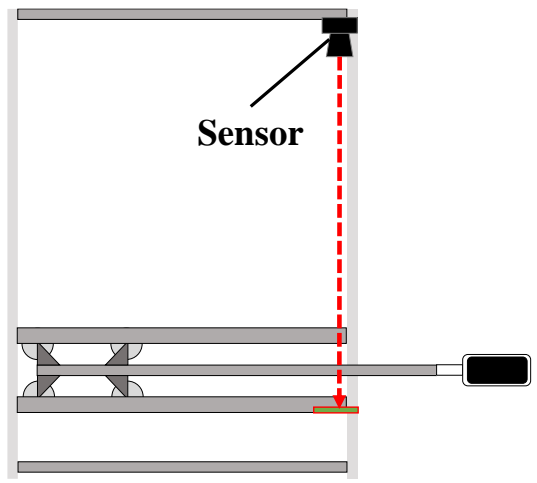

(b) Sensor.

Fig. 4. Moves up and down.

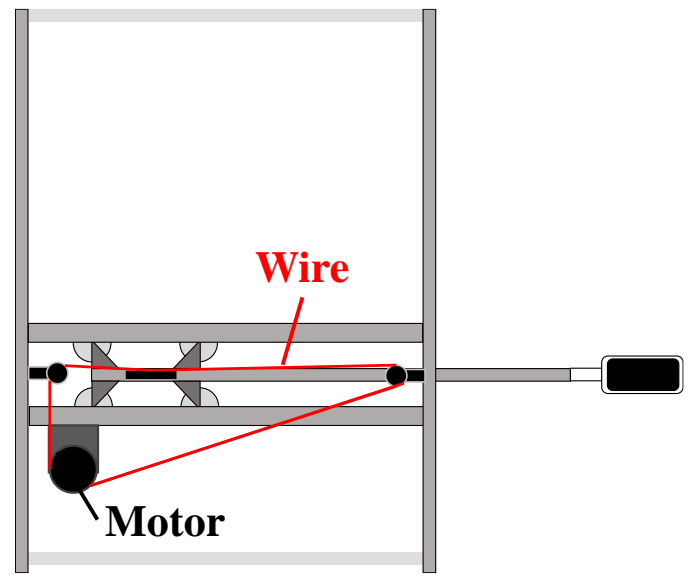

(a) Wire and motor.

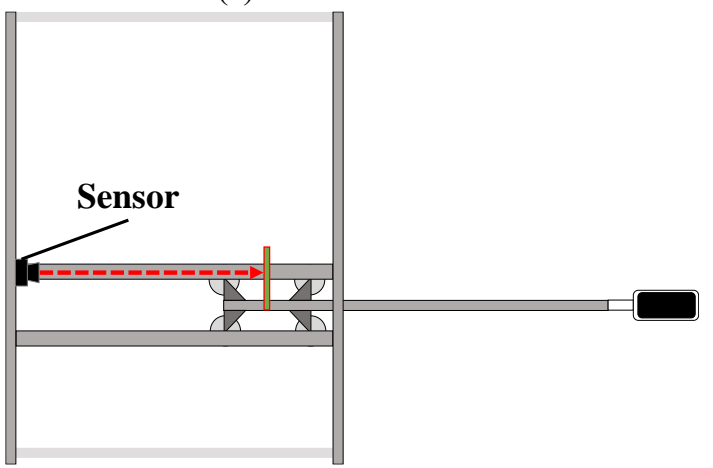

(b) Sensor.

Fig. 5. Extend.

clothes, the brush is shrunk to finish the operation.

The mechanism of each axis is shown in the sections of $2.1 \sim 2.3$.

\subsection{Axis to hold clothes}

It is necessary to adhere the brushes to the clothes and to fix the clothes. In order to remove dust from clothes in this study, we adhere and fix by sandwiching the clothes between the two brushes. The mechanism to perform the above operation is explained in the following illustrations. As shown in Fig. 3(a), a motor and a wire are attached to move the shaft left and right in order to pinch the clothes. Furthermore, a sensor is used to control the state of the axis as shown in Fig. 3(b).

\subsection{Axis to move the brush up and down}

The brush needs to be moved up and down in order to collect the dust from the clothes. The mechanism for moving the shaft up and down is explained in the following illustrations. To move the shaft up and down, attach the motor and the wires as shown in Fig. 4(a). Furthermore, a sensor is attached to control the state of the axis as shown in Fig. 4(b). 


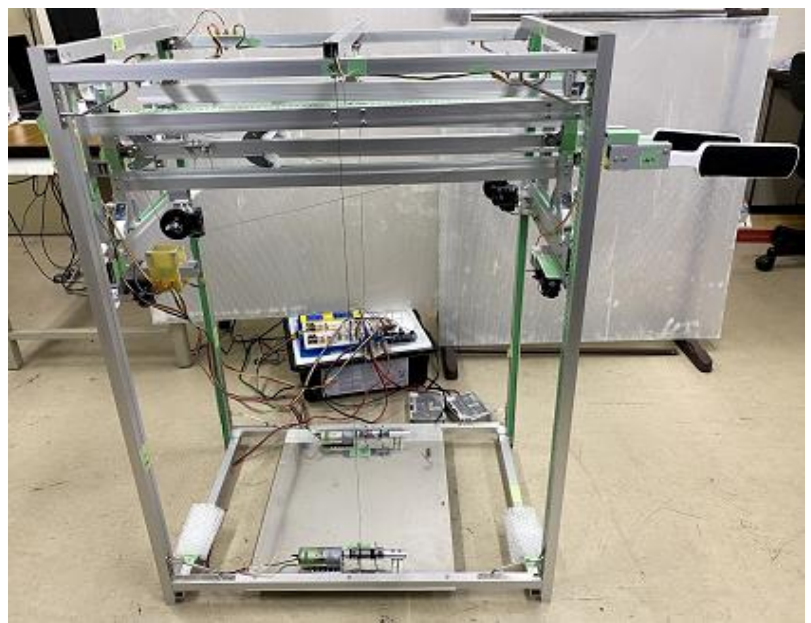

(a) Front view.

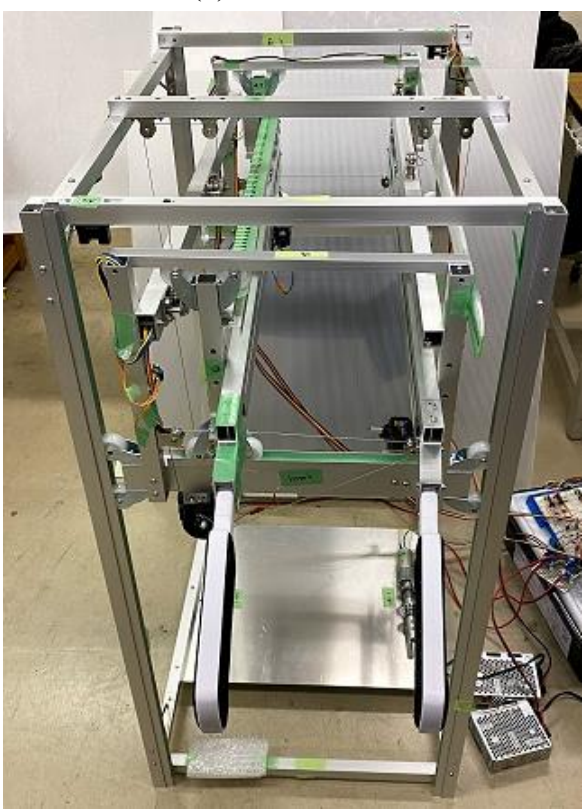

(b) Side view.

Fig. 6. Robot.

\subsection{Axis to extend the brush}

The shoulder width of the clothes is longer than the length of the brush. Thus, to get the dust off the entire clothes, need to brush it several time. In this paper, we realize remove dust from the entire clothes by stretching the brush several times. The mechanism to perform the above operation is explained using the next illustrations. To extend the shaft, attach the motor and the wires as shown in Fig. 5(a). In addition, attach the sensor shown in Fig. 5(b) to control the state of the axis.

\section{Robot of prototype}

In this section, we describe the prototype of the robot

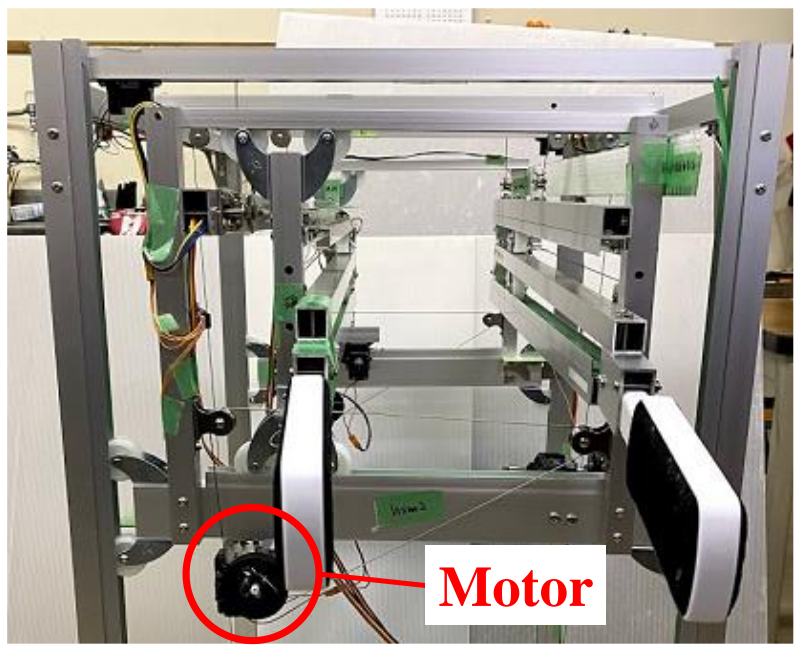

(a) Drive unit 1 .

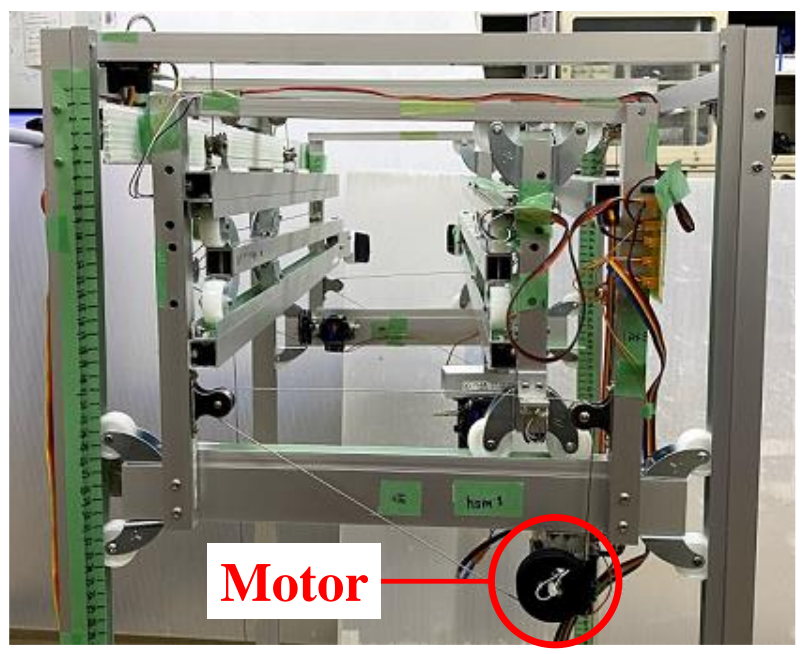

(b) Drive unit 2.

Fig. 7. Drive units.

described in Chapter 2. The overall view of the robot is shown in Fig. 6. As shown in Fig. 6, the robot is box-shaped, but the arm with a brush (Copa Corporation, MUGENBRUSH) sticks out to pinch clothes. The size of the box part of this robot is about $740 \mathrm{~W} \times 495 \mathrm{D} \times 1000 \mathrm{H}$ $(\mathrm{mm})$. The length of the protruding arm is $380 \sim 830(\mathrm{~mm})$. The robot automatically removes dust from clothes hung on the hanger rack.

The details of the three axes described in Chapter 2 are explained in the sections of $3.1 \sim 3.3$.

\subsection{Axis to hold clothes}

A photo of the pinching axis is shown in Fig. 7.

As shown in Fig. 7, only one of the brushes has a drive unit on each side. The two drive units are called the drive unit 1 in Fig. 7(a) and the drive unit 2 in Fig. 7(b), respectively. The two drive units are driven simultaneously to pinch the 


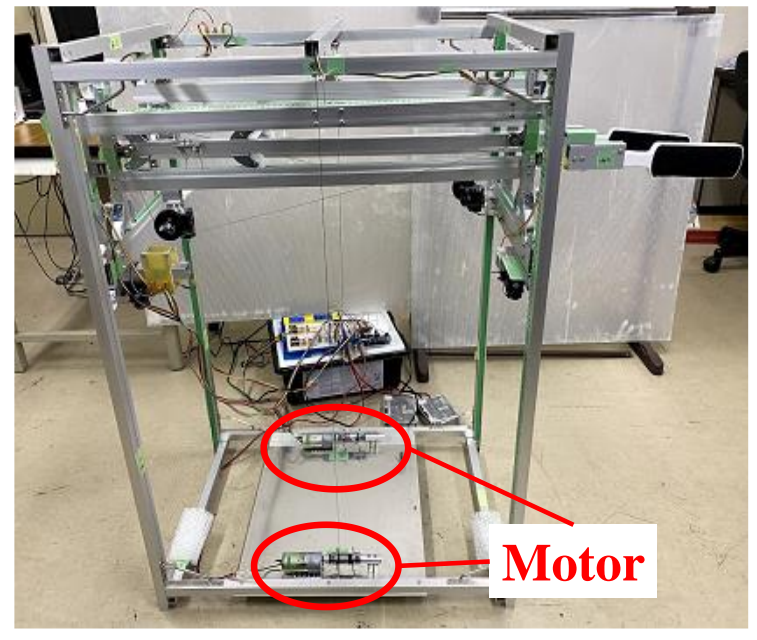

Fig. 8. Moves up and down.

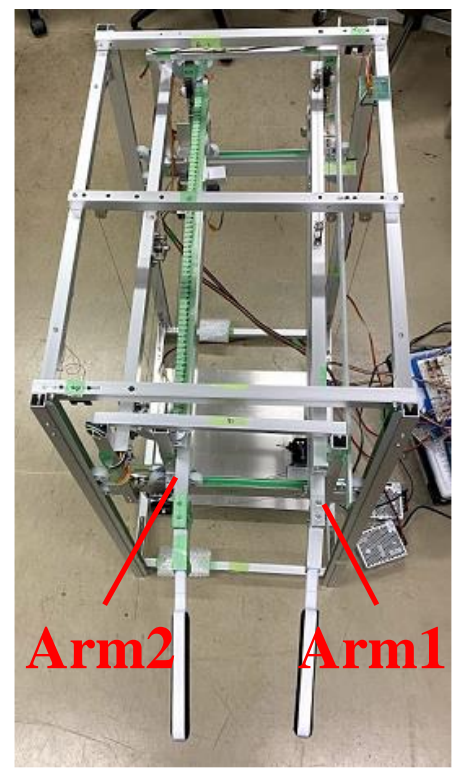

Fig. 9. Arm.

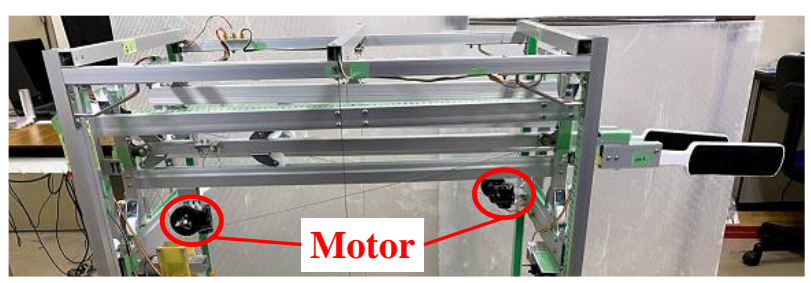

Fig. 10. Extend.

clothes. The brushes are driven up to $160 \mathrm{~mm}$ in the pinching direction. This motion is achieved by winding the wire using a motor (TAMIYA, 4-Speed Worm Gearbox H.E.) and pulleys. The motor is run using a $5(\mathrm{~V}) \mathrm{DC}$ power supply, and the drive direction is controlled using a motor driver (Toshiba, TA7291P). In addition, a distance sensor (Sharp,
Table 1. Press.

\begin{tabular}{|c|c|c|}
\hline \multirow{2}{*}{ Position } & \multicolumn{2}{|c|}{ Output voltage(V) } \\
\cline { 2 - 3 } & Drive1 & Drive2 \\
\hline open & 2.2 & 2.2 \\
\hline close & 1.4 & 1.5 \\
\hline
\end{tabular}

Table 2. Moves up and down.

\begin{tabular}{|c|c|c|}
\hline \multirow{2}{*}{ Position } & \multicolumn{2}{|c|}{ Output voltage(V) } \\
\cline { 2 - 3 } & arm1 & arm2 \\
\hline Topmost & 2.2 & 1.7 \\
\hline Lowermost & 1.1 & 0.75 \\
\hline
\end{tabular}

GP2Y0E03) is installed in each of the two driving sections, and the width of the sandwich is controlled using an arduino.

\subsection{Axis to move the brush up and down}

Fig. 8 shows the mechanism for moving the brush up and down in the prototype. The two brushes are moved up and down by simultaneously driving the two mechanism for extending brushes as shown Fig. 8. Fig. 9 shows the top view of the arms with the brushes attached. The arms are called Arm 1 and Arm 2 respectively, as shown in Fig. 9. The brushes are driven up and down the maximum of $520(\mathrm{~mm})$. The motor (TAMIYA, AO-8037 Geared Motor 540K300) and the wires are used for this movement. The motor runs on a $12(\mathrm{~V})$ DC power supply and winds the wire with a pipe. The motor driver (Pololu, VNH3SP30) is used to control the drive direction. In addition, a distance sensor (Sharp, GP2Y0A02) is installed in each of the two drive sections, and the height at which it is moved can be controlled using an arduino.

\subsection{Axis to extend the brush}

Fig. 10 shows the mechanism for extending the brushes of the prototype as in Section 3.2, we distinguish the arms to be driven as in Fig. 9. The brushes are driven the maximum of $450(\mathrm{~mm})$. This movement is achieved by using a motor (TAMIYA, 4-Speed Worm Gearbox H.E.) and a pulley to wind the wire. The motor is run using a $5(\mathrm{~V})$ DC power supply, and the drive method is controlled using a motor driver (Toshiba, TA7291P). In addition, the axis to be extended can be seen by the distance sensor (Sharp, GP2Y0A21YK0F), and the distance to extend the brush can be controlled using the arduino. 
Table 3. Extend.

\begin{tabular}{|c|c|c|c|c|}
\hline \multirow{2}{*}{ Position } & \multicolumn{2}{|c|}{ Distance $(\mathrm{mm})$} & \multicolumn{2}{c|}{ Output voltage(V) } \\
\cline { 2 - 5 } & arm1 & arm2 & arm1 & arm2 \\
\hline Initial & 140 & 110 & 1.8 & 2.2 \\
\hline 1 & 280 & 250 & 1.0 & 1.1 \\
\hline 2 & 420 & 390 & 0.8 & 0.7 \\
\hline 3 & 560 & 530 & 0.7 & 0.6 \\
\hline
\end{tabular}

\section{Experiment}

In this study, the entire clothes are dusted repeatedly by controlling three axes: the axis that holds the clothes, the axis that moves the brush up and down, and the axis that extends the brush. After confirming the operation of each axis, we briefly performed the operation of the entire system. The outputs of all the sensors used in the prototype were averaged 50 times to reduce an error.

The experiments conducted in this study are shown in the sections of $4.1 \sim 4.4$.

\subsection{Axis to hold clothes}

We checked the output of the sensor and determined the threshold value to control each axis.

We checked the value of the output voltage of the sensor when the clothes were pinched and when it was open. Table 1 shows the outputs when the arm was open, and when it was closed. According to Table 1, these were used as the threshold values, and when both the left and the right drives were lower than each threshold value, both drives were moved simultaneously. When only one of them reached the threshold when the arm was closed, only the drive unit that had not reached the threshold was moved.

\subsection{Axis to move the brush up and down}

In this experiment, a hanger rack of $840 \mathrm{~W} \times 1000 \mathrm{H}$ $(\mathrm{mm})$ and a hanger of $400 \mathrm{~W} \times 210 \mathrm{H}(\mathrm{mm})$ was used. When a hanger was hung on the hanger rack, the top of the clothes was considered to be just below the hanger. At that time, the top of the clothes was $800(\mathrm{~mm})$ from the ground. Therefore, we assumed that the moving part needed to be moved within the range of $300-800 \mathrm{~mm}$ from the ground in order to remove dust from the top to the bottom of the clothes. We checked each output voltage when the arm was at the top and at the bottom. The output voltages when the arm is at the top and at the bottom are shown in Table 2. Table 2 says, the arm was moved up and down from the top of the clothes to the bottom, using this as the threshold. When neither of them reached the threshold, the left and the right drives were moved. When only one of them reached the threshold, only the drive unit of the one that had not reached the threshold was moved.

\subsection{Axis to extend the brush}

The width of the brush was $150(\mathrm{~mm})$ and the width of the hanger was $400(\mathrm{~mm})$ from the section 4.2. The arm was moved to the clothes side three times every $140(\mathrm{~mm})$ in order to remove dusts the entire clothes. Table 3 shows the position of each arm and the output voltage when moved every $140(\mathrm{~mm})$. Using the values in Table 3, the arm was moved from the initial position to position 3. After the arm reached position 3 , it was returned to the initial position.

\subsection{Check the entire system}

An experiment was conducted to remove dust from clothes on a hanger rack using a prototype robot. A 100(\%) polyester, small size short pants was used as a sample of the clothes. The hangers were fixed to the hanger rack with curing tape to prevent shaking. Dust was collected and evenly adhered to the short pants, and the experiment was conducted. The operation of the prototype during the experiment is shown in Fig. 11. First, the arms were opened and raised to the top of the short pants as shown in Fig. 11(a) and (b). Next, as shown in Fig. 11(c) and (d), the short pants were pinched and the arm was lowered to remove dust. To remove every dust from the short pants, the arm was extended as shown in Fig. 11(e) and (f), and the above operation was repeated to remove the dust from the short pants. As shown in Fig. 11(g) and (h), the entire short pants were removed dusts, the arm was closed and retracted.

As a result of the experiment, the operating time of the prototype was about 3 minutes. Fig. 12 shows the short pants before and after the robot removed the dust. From Fig. 12, it was confirmed that the dust on the clothes was removed. 


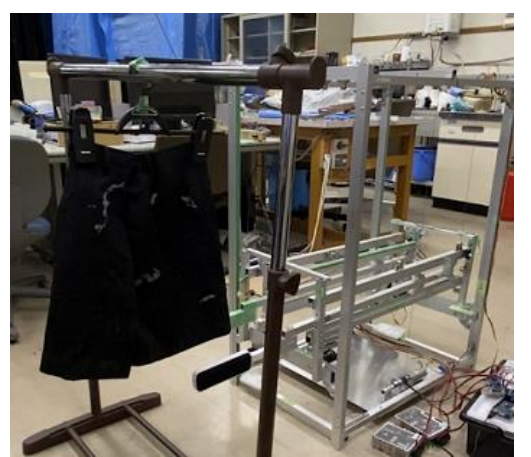

(a) Step 1 .

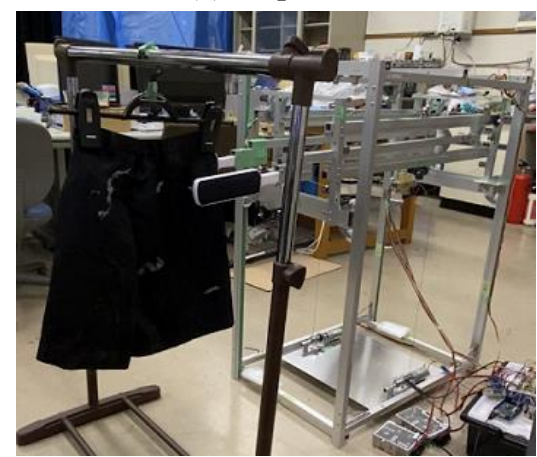

(b) Step 2 .

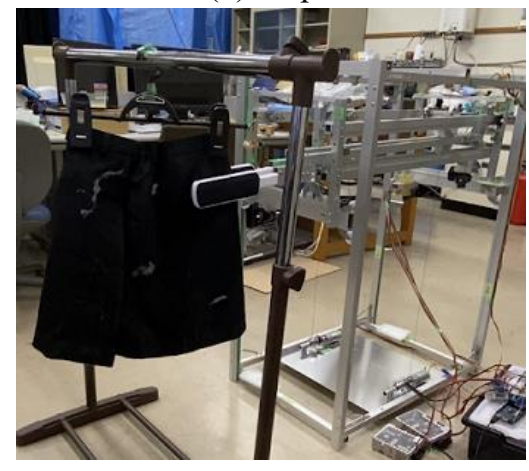

(c) Step 3 .

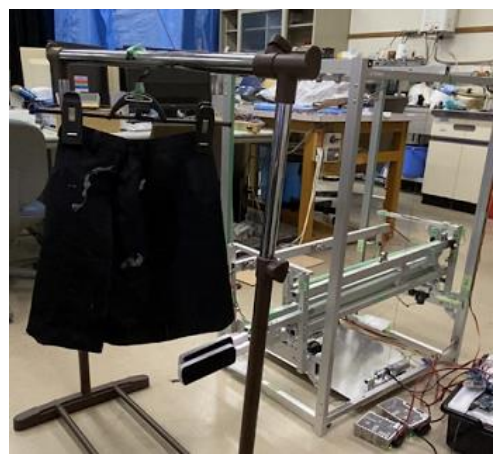

(d) Step 4

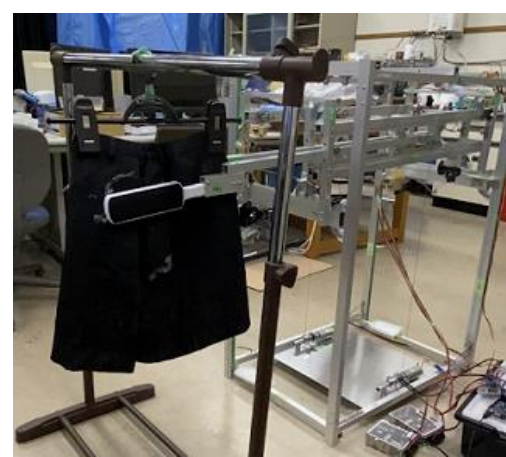

(e) Step 5 .

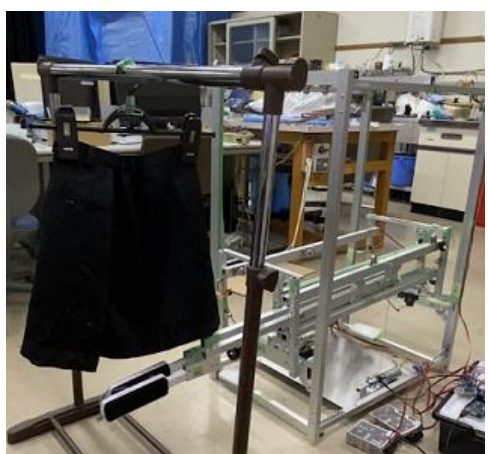

(f) Step 6 .

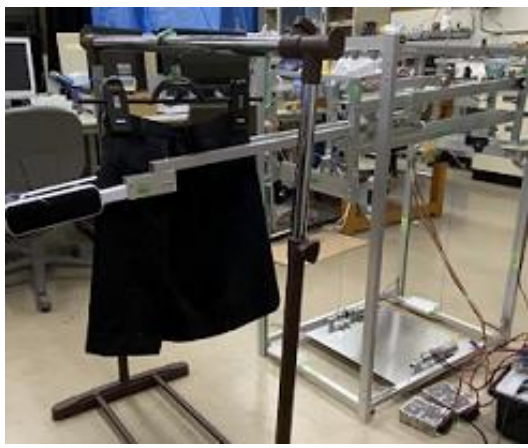

(g) Step 7.

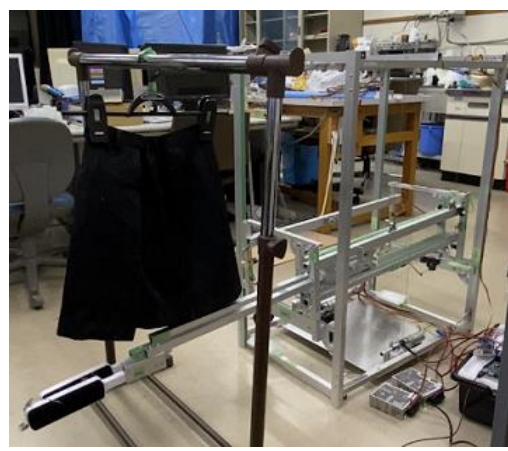

(h) Step 8 .

Fig. 11. Experiment

\section{Consideration}

In this study, we developed a robot that removes dust from clothes on hangers by controlling three axes: the axis that grips the clothes, the axis that moves the brush up and down, and the axis that extends the brush. It was confirmed that this robot could remove most of the dust. This robot can automatically remove dust by pressing the start button. The user only needs to hang the clothes on the hanger rack and collect the clothes after the robot's work. Thus, it can reduce the burden and save time. 


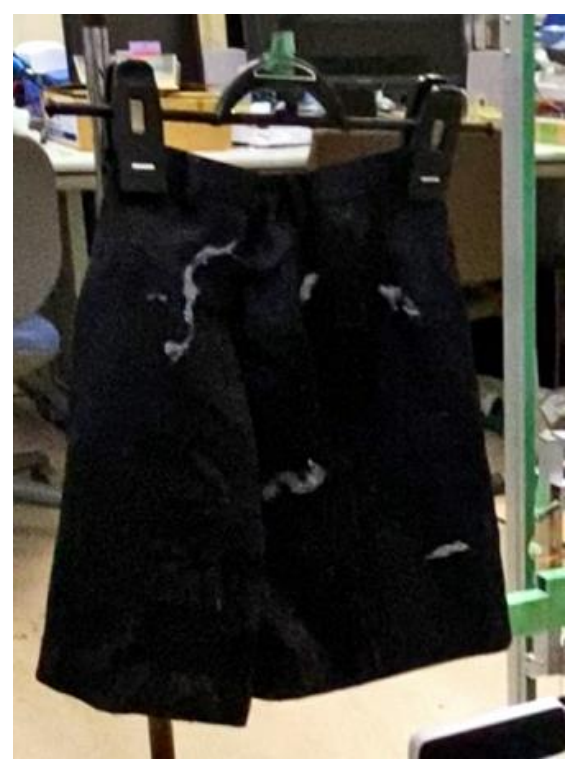

(a) Before the test.

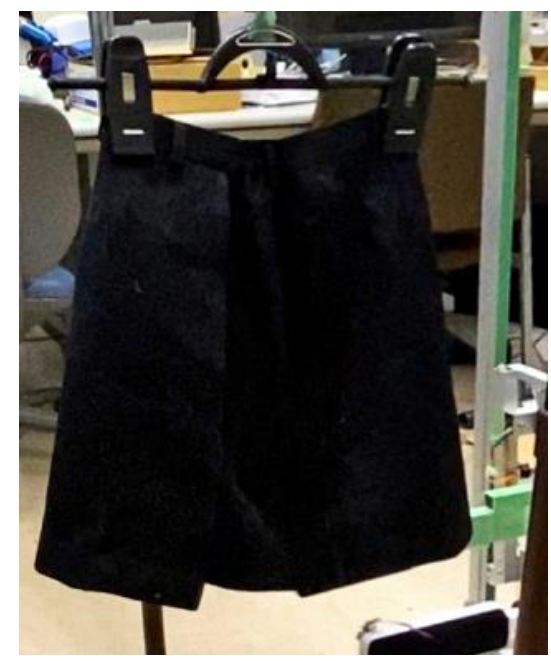

(b) After the test.

Fig. 12. Result.

The future work includes improving the stability of the axis and recognizing the clothes in image processing. In addition, the system would be more convenient if it were continuously used for multiple clothes in a row.

\section{References}

(1) LSI Medience Corporation : "Allergy Information", https://www.medience.co.jp/allergy/koumoku.html, 2021/01/20

(2) Tuduki Yasunobu and Narita Mituo : "Twin cases of anaphylaxis caused by ticks in okonomiyaki flour", Journal of the Japanese Society of Internal Medicine, Vol. 104, No. 5, pp.986-990, 2015

(3) Muko Ryo, Matsuda Hiroshi and Tanaka Akane : "Animal model of skin allergy - Screening and evaluation of functional substances", Functional Food Research, Vol. 14, pp.23-29, 2018

(4) Tameny : "The number one requirement for a marriage partner is someone with a sense of cleanliness.", https://www.p-a.jp/research/report 144.html, 2021/01/20

(5) Statistics Bureau, Ministry of Finance :

"Prefecture, Number of general households by number of persons in household and number of persons in household by type of household", https://www.stat.go.jp/data/nihon/02.html, 2021/01/20

(6) Fujii Syouichi, Konno Keiichi, Kimura Kikuji and Yoshizawa Susumu : "3013 The amount of dust generated indoors", Proceedings of the Architectural Institute of Japan, Extra Issue, Extra Edition, Academic Abstracts, Vol. 41, p. 395-, 1966

(7) Pet Food Association of Japan : "National Survey of Dog and Cat Ownership - Key Indicators and Summary -”, $\quad$ https://petfood.or.jp/data/chart2020/3.pdf, 2021/01/20

(8) Yamada Masaru : "Hair Loss in Labrador Retrievers", Japan Assistance Dog Science Research, Vol. 2, No. 1, pp.36-40, 2008 